\title{
Targeted PLGA-Microparticles as a Novel Concept for Treatment of Lactose Intolerance
}

\author{
X. WANG ${ }^{\#}$, G. RATZINGER , M. WIRTH, F. GABOR
}

Department of Pharmaceutical Technology and Biopharmaceutics, Faculty of Life Sciences, University of Vienna, Vienna, Austria.

\# These authors equally contributed to this work.

E-mails: xueyan.wang@univie.ac.at (X.Wang), franz.gabor@univie.ac.at (F. Gabor)

Sci Pharm. 2010; 78: 559 doi:10.3797/scipharm.cespt.8.LDD05

Background: Lactose intolerance is the inability to metabolize lactose because of the absence of the enzyme lactase. It is estimated that $75-90 \%$ of birth lactase levels are lost by most people after weaning. The prevalence of lactase deficiency ranges widely with the ethnic background from 2-15\% among Northern Europeans to $95-100 \%$ among Asians [1]. Nowadays, lactose intolerance is usually controlled by strict adherence to a loactose-free or lactose-reduced diet. Moreover, as an alternative, capsules or tablets containing microbial-derived ß-galactosidase are available. However, this treatment is inconvenient for patients since these formulations have to be administered immediately before or together with lactose-containing diet because of their short-acting effect.

Aim: Therefore, the present work is aimed to develop an innovative long-acting peroral formulation for the treatment of lactose intolerance.

Methods: Biodegradable and biocompatibale polymeric microcarriers $(2.78 \pm 1.05 \mu \mathrm{m}$ in diameter) were manufactured from poly(D,L-lactide-coglycolide) (PLGA) using spray-drying. They were functionalized with $\beta$-galactosidase from Kluyveromyces lactis and targeted with wheat germ agglutinin (WGA), which might prolong the residence time of particles in the small intestine. The particle-bound enzyme activity, the mucoadhesive as well as the cytoadhesive properties were assessed.

Results: The highest particle-bound enzyme activity (1470 U ß-galactosidase per gram PLGA) was obtained with hexamethylene diamine as a spacer using carbodiimide method representing a 6-fold increase as compared to particles without spacer. Surface immobilisation of WGA enhanced considerably the particle binding to porcine mucin layer (mucoadhesion) and Caco-2 cell monolayers (cytoadhesion).

Conclusions: PLGA-microparticles, surface-modified with active ß-galactosidase as enzyme substitute and WGA as a targeter, are able to bind to enterocytes and thereby to prolong the intestinal residence time. It is a promising approach towards a promising approach towards a more convenient therapy of lactose deficiency and intolerance.

[1] Harrington LK, Mayberry JF. A re-appraisal of lactose intolerance. Int J Clin Pract. 2008; 62: 15411546. doi:10.1111/j.1742-1241.2008.01834.x 